\title{
The role of SU(3)-flavour symmetry breaking in B-meson decay constants from Lattice QCD
}

\author{
Sophie Hollitt*, Paul Jackson, Ross Young, and James Zanotti \\ CSSM and CoEPP, Department of Physics, \\ The University of Adelaide, Adelaide SA 5005, Australia \\ E-mail: sophie.hollitteadelaide.edu.au
}

\begin{abstract}
$B$-physics is currently facing a number of puzzles, and additional precision data - and precision calculations - will be required to pick these apart. In particular, as the Belle II detector approaches completion, there is increasing need for lattice calculations related to CKM matrix elements and to important $B$ branching ratios such as $\mathscr{B}(B \rightarrow \tau \nu)$.

We present early results for $f_{B}$ and $f_{B_{S}}$ from the CSSM/QCDSF/UKQCD collaboration on a set of lattices with a fixed volume $32^{3} \times 64$ and lattice spacing $a=0.074 \mathrm{fm}$. By varying the $u, d$,s quark masses while holding their average value constant, we are able to reliably control the SU(3)flavour breaking effects.
\end{abstract}

The 26th International Nuclear Physics Conference

11-16 September, 2016

Adelaide, Australia

${ }^{*}$ Speaker. 


\section{Introduction}

Results from Belle and BABAR were crucial for our understanding of $B$-physics and the unitarity in the CKM matrix, but these results have also left us with a number of puzzles where further understanding of QCD (and of our detectors) is required. As the Belle II experiment [1] approaches its first science run and $\mathrm{LHCb}$ continues to increase statistics, the pressure is on to improve errors on theoretical and lattice calculations ahead of future improvements to experimental precision. We choose to focus on the $B$-meson decay constants $f_{B}$ and $f_{B_{s}}$ which are crucial to Standard Model calculations of the branching ratio $\mathscr{B}(B \rightarrow \tau \nu)$, though the $f_{B}$ and $f_{B_{s}}$ also appear in calculations of CKM matrix elements $\left|V_{t b}\right|$ and $\left|V_{t s}\right|$ from measurements of CP violation in $B^{0}$ and $\bar{B}^{0}$ mesons.

The Flavour Lattice Averaging Group (FLAG) reviews [2,3] indicate that there are several groups working on $f_{B}$ and related $B$ meson observables with an eye toward improved precision [49]. As a companion to this body of existing work, we investigate $f_{B}$ and $f_{B_{s}}$ with a focus on $\mathrm{SU}(3)$ symmetry breaking effects by choosing light and strange quarks with a constant average mass $\bar{m}$. This methodology comes from the UKQCD/QCDSF group and we follow a similar method in our $f_{B} / f_{B_{s}}$ study as in their related study of $f_{\pi}$ and $f_{K}$. [10]

\section{Simulation Details}

\subsection{Treatment of light and strange quarks}

When extrapolating to the physical point using multiple lattice ensembles with different quark masses, many groups will choose the strange quark mass $m_{s}$ to be constant. We instead follow the UKQCD/QCDSF process for choosing the masses of light and strange quarks in a $2+1$ formalism [10]. The value of $\bar{m}=\frac{1}{3}\left(2 m_{l}+m_{s}\right)$ is kept constant to control symmetry breaking and remove effects of $\mathscr{O}(\delta m)$. In fact, all flavour singlet quantities are only affected by SU(3)-flavour breaking effects at $\mathscr{O}\left((\delta m)^{2}\right)$, and have been shown to stay approximately constant from the $\mathrm{SU}(3)$ symmetric point to the physical point. [10]

For the ensembles of lattice configurations used in this work, there is a mixture of ensembles where $\bar{m}$ is equal to the physical value of $\bar{m}$ and ensembles where $\bar{m}$ has a slightly different value. The relationship between $m_{l}$ and $m_{s}$ (or equivalently, the relationship between the pion and kaon masses) for different ensembles at $a=0.0074 \mathrm{fm}$ [11] is shown graphically in Figure 1 (inset) and also displayed in Table 1.

\subsection{Bottom quarks}

We generate bottom quarks using the anisotropic clover-improved action [7]

$$
S_{\text {lat }}=a^{4} \sum_{x, x^{\prime}} \bar{\psi}\left(x^{\prime}\right)\left(m_{0}+\gamma_{0} D_{0}+\zeta \vec{\gamma} \cdot \vec{D}-\frac{a}{2}\left(D^{0}\right)^{2}-\frac{a}{2} \zeta(\vec{D})^{2}+\sum_{\mu, v} \frac{i a}{4} c_{P} \sigma_{\mu v} F_{\mu v}\right)_{x, x^{\prime}} \psi(x)
$$

and tune $m_{0}, c_{P}$ and $\zeta$ to specify the mass, hyperfine splitting, and dispersion relation of the generated $B(*)$ or $B(*)_{s}$ mesons. This is a variant of the 'Fermilab action' or 'RHQ action' $[12,13]$. We choose the 'best' tuning by considering a flavour singlet $B$-meson $X_{B}=\frac{1}{3}\left(2 B_{l}+B_{s}\right)$ and selecting 
the tuning parameters such that our calculated $B$ and $B_{s}$ mesons combine to create an $X_{B}$ matching $X_{B}$ for the physical $B$ and $B_{s}$.

In practice, uncertainties on the measured mass, splitting, and dispersion relation also result in uncertainties in the values of $m_{0}, c_{P}$ and $\zeta$ corresponding to the 'best' tuned $B$ meson. We choose to always generate multiple $b$-quarks per lattice ensemble and interpolate to the 'best' $B$, rather than generating only one 'best' $b$-quark after completing the tuning process. This allows us to use the same set of seven $b$-quarks for each ensemble with the same lattice spacing and volume.

\subsection{Additional Information}

In this work, we use multiple ensembles of $\sim 800$ gauge field configurations with $2+1$ flavours of non-perturbatively $\mathscr{O}(a)$ improved Wilson fermions. Further details of the configurations used in each ensemble are shown in Table 1 and Table 2.

\begin{tabular}{|c|c|c|c|c|c|c|}
\hline$\left(\kappa_{l}, \kappa_{s}\right)$ & $\begin{array}{l}m_{\pi} \\
(\mathrm{MeV})\end{array}$ & $\begin{array}{l}m_{K} \\
(\mathrm{MeV})\end{array}$ & $\begin{array}{l}\# \text { configs } \\
\text { used }\end{array}$ & $m_{0}$ & $c_{P}$ & $\zeta$ \\
\hline$(0.12090,0.12090)$ & 465 & 465 & 778 & $2.80 \pm 0.13$ & $3.60 \pm 0.34$ & $1.30 \pm 0.11$ \\
\hline$(0.12104,0.12062)$ & 360 & 505 & 758 & $2.65 \pm 0.11$ & $3.19 \pm 0.29$ & $1.37 \pm 0.11$ \\
\hline$(0.121095,0.120512)$ & 310 & 520 & 380 & $2.98 \pm 0.22$ & $4.03 \pm 0.57$ & $1.21 \pm 0.16$ \\
\hline$(0.12095,0.12095)$ & 400 & 400 & 400 & $2.69 \pm 0.15$ & $3.29 \pm 0.39$ & $1.50 \pm 0.14$ \\
\hline$(0.12104,0.12077)$ & 330 & 435 & 786 & $2.82 \pm 0.13$ & $3.59 \pm 0.34$ & $1.38 \pm 0.10$ \\
\hline
\end{tabular}

Table 1: Lattice configurations and tuning results for ensembles with $V=32^{3} \times 64, a=0.074 \mathrm{fm}$, and $\beta=5.5$ used in $\mathrm{SU}(3)$ breaking calculations

\begin{tabular}{|c|c|c|c|c|c|c|c|}
\hline $\mathrm{V}$ & $a(\mathrm{fm})$ & $\beta$ & $\kappa_{l}=\kappa_{s}$ & $\begin{array}{l}\# \text { configs } \\
\text { used }\end{array}$ & $m_{0}$ & $c_{P}$ & $\zeta$ \\
\hline $24^{3} \times 48$ & 0.0818 & 5.4 & 0.11993 & 808 & $3.90 \pm 0.32$ & $4.64 \pm 0.91$ & $1.33 \pm 0.24$ \\
\hline $32^{3} \times 64$ & 0.074 & 5.5 & 0.120900 & 778 & $2.80 \pm 0.13$ & $3.60 \pm 0.34$ & $1.30 \pm 0.11$ \\
\hline $32^{3} \times 64$ & 0.0684 & 5.65 & 0.122005 & 410 & $2.74 \pm 0.18$ & $4.34 \pm 0.48$ & $1.13 \pm 0.12$ \\
\hline
\end{tabular}

Table 2: Lattice configurations and tuning results for additional ensembles at the SU(3) symmetric point

The source locations for quarks are randomised to reduce correlations between neighbouring configurations in the ensemble. Future work will include either more configurations for each lattice ensemble where additional configurations are available, or additional source locations on the same configurations to increase statistics.

\section{Calculating $f_{B}$ on the lattice}

The decay constant $f_{B}$ is calculated from its lattice counterpart $\Phi_{B}$ via the equation

$$
f_{B}=\frac{\hbar c}{a} Z_{\Phi} \Phi_{B}
$$


where $\Phi_{B}$ is calculated from two-point correlators for Axial and Pseudoscalar operators:

$$
\Phi_{B}=-\frac{\sqrt{2 M_{B}} \mathscr{C}_{A P}}{\mathscr{C}_{P P}}, \quad \mathscr{C}_{A P}=\frac{\left\langle\Omega\left|A_{4}\right| B\right\rangle\langle B|P| \Omega\rangle}{2 M_{B}}, \quad \mathscr{C}_{P P}=\frac{\langle\Omega|P| B\rangle\langle B|P| \Omega\rangle}{2 M_{B}}
$$

and $Z_{\Phi}$ is calculated:

$$
Z_{\Phi}=\rho_{A}^{b l} \sqrt{Z_{V}^{b b} Z_{V}^{l l}}
$$

This formulation of $\Phi_{B}$ is equivalent to that used in [6], which does not include the factor $2 m$ in the correlators.

In practice, $Z_{V}^{b b}$ has been calculated using a light spectator quark in the three-point correlator, and we use $\rho_{\text {lat }}^{b l}=1$ for these early calculations of $f_{B} . f_{B}$ can also be improved by letting $\Phi_{B}$ go to $\Phi_{B}^{0}+c_{A} \Phi_{B}^{1}$ using an improvement coefficient $c_{A}$, but this has not yet been explored at the time of this work.

\section{Results}

\subsection{SU(3) symmetry breaking in $f_{B}$ and $f_{B_{s}}$}

Following the procedure used in the light quark sector [14], we plot $f_{B}$ and $f_{B_{s}}$ relative to the average decay constant $f_{B_{X}}=\frac{1}{3}\left(2 * f_{B}+f_{B_{s}}\right)$ in order to cancel out some errors and look for SU(3)flavour breaking behaviour. If $\mathrm{SU}(3)$ breaking of the lighter quarks is the main effect, we expect a linear fit for $f_{B} / f_{B_{X}}$ against $m_{\pi}^{2} / m_{X}^{2}$ as was observed for $f_{\pi}$ and $f_{K}$ in [14]. These early results with a linear fit show good agreement with the FLAG2013 average of $N=2+1$ flavour calculations. [2]
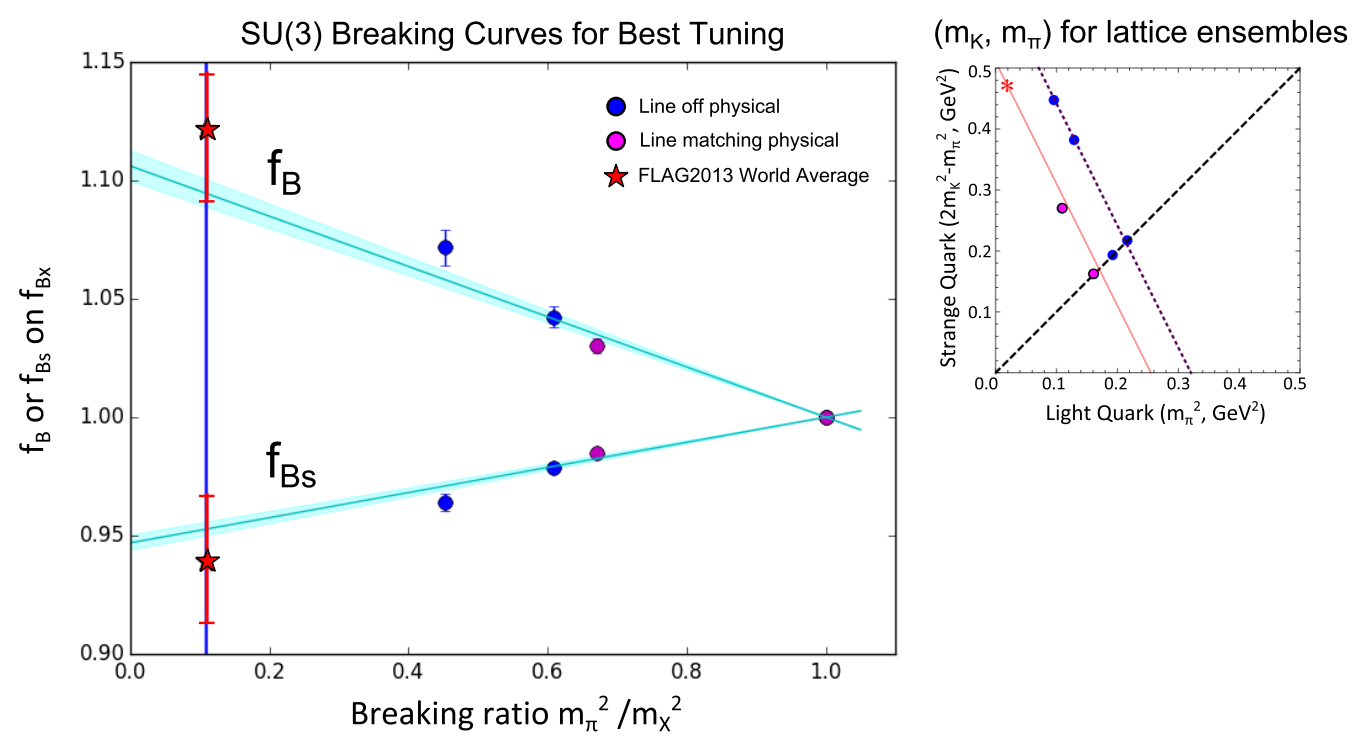

Figure 1: (Main Graph): Calculated values for $f_{B}$ and $f_{B_{S}}$ are plotted relative to the average decay constant $f_{B_{X}}=\frac{1}{3}\left(2 * f_{B}+f_{B_{s}}\right)$. (Inset): Legend of location of lattice ensembles on a plane representing the strange and light quark masses relative to the physical point, plot taken from [15] 


\subsection{Toward predictions for $f_{B}$ and $f_{B_{s}}$}

It is crucial to control as many sources of uncertainty as possible in order to have competitive calculated values for $f_{B}$ and $f_{B_{s}}$. As part of an investigation of lattice artefacts, we examine the way that the normalisation factor $Z_{\Phi}$ changes as the lattice volume and lattice spacing are varied. The effect of this normalisation on $f_{B}$ is shown in Figure 2, while the changes in the components $\sqrt{Z_{V}^{b b}}$ and $\sqrt{Z_{V}^{l l}}$ of $Z_{\Phi}$ are plotted against lattice spacing $a^{2}$ in Figure 3.
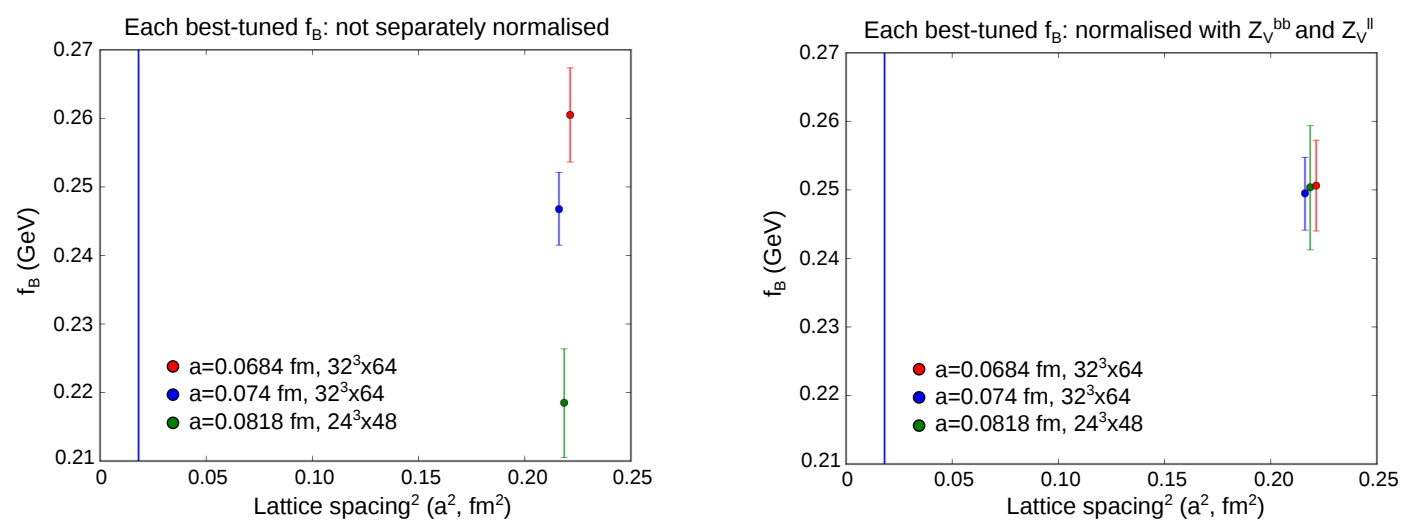

Figure 2: (Left) $f_{B}$, where the same $Z_{\Phi}$ value is used regardless of lattice spacing/lattice volume (Right) $f_{B}$, where $Z_{\Phi}$ is calculated for each lattice ensemble individually.
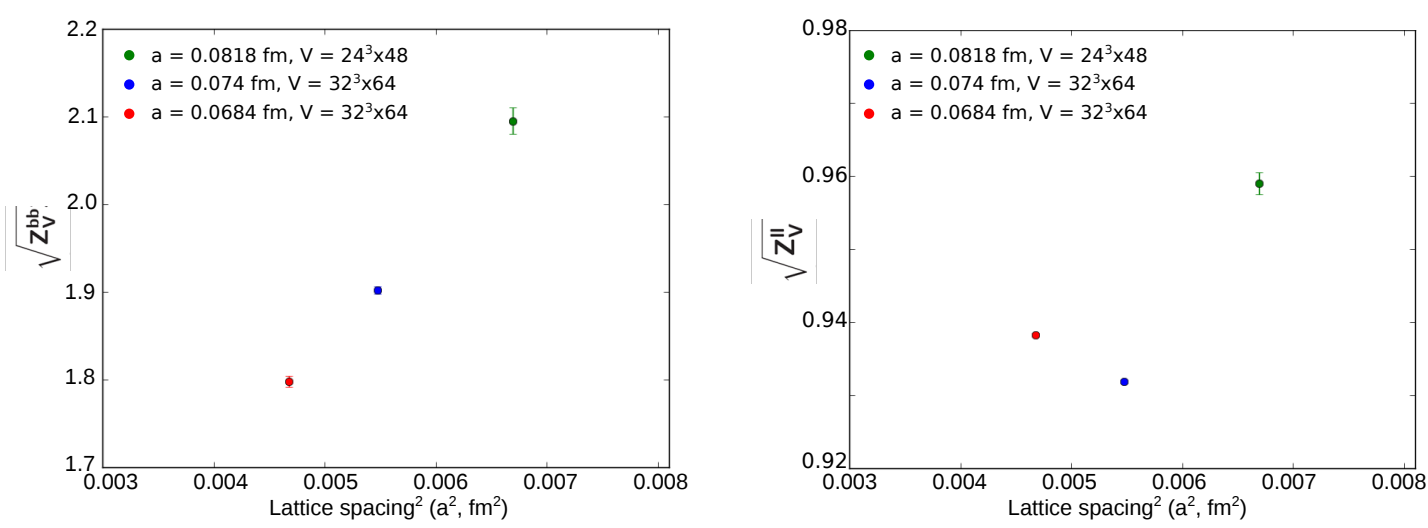

Figure 3: (Left) Normalisation factor $\sqrt{Z_{V}^{b b}}$ against lattice spacing squared. The relationship between the two is approximately linear. (Right) Normalisation factor $\sqrt{Z_{V}^{l l}}$ against lattice spacing squared. $Z_{V}^{l l}$ appears to be affected most strongly by the lattice volume.

While $\sqrt{Z_{V}^{b b}}$ changes linearly with $a^{2}$ and appears to be unaffected by the lattice volume, the value of $Z_{V}^{l l}$ is significantly different for the $24^{3} \times 48$ and $32^{3} \times 64$ lattices. We suspect that this difference is due to discretisation errors on the small $24^{3} \times 48$ lattices, but this remains to be 
checked in future work. Future work will also involve $48^{3} \times 96$ lattices for additional comparison at $\beta=5.65$ as well as at finer lattice spacings $(\beta=5.8,5.95)$.

\section{Conclusion}

We have presented preliminary results for $f_{B}$ and $f_{B_{s}}$ with controlled SU(3) symmetry breaking by controlling the way light and strange quark masses are chosen. Investigation of systematic lattice discretisation effects is underway, and we look forward to further progress toward calculations of $f_{B}$ and $f_{B_{s}}$ with competitive errors.

\section{Acknowledgments}

The numerical configuration generation was performed using the BQCD lattice QCD program [16], on the IBM BlueGeneQ using DIRAC 2 resources (EPCC, Edinburgh, UK), the BlueGene P and Q at NIC (Jülich, Germany) and the Cray XC30 at HLRN (Berlin-Hannover, Germany). Some of the simulations were undertaken using resources awarded at the NCI National Facility in Canberra, Australia, and the iVEC facilities at the Pawsey Supercomputing Centre. These resources are provided through the National Computational Merit Allocation Scheme and the University of Adelaide Partner Share supported by the Australian Government. This work was supported in part through supercomputing resources provided by the Phoenix HPC service at the University of Adelaide. The BlueGene codes were optimised using Bagel [17]. The Chroma software library [18], was used in the data analysis. This investigation has been supported by the Australian Research Council under grants FT120100821, FT100100005, FT130100018 and DP140103067 (RDY and JMZ).

\section{References}

[1] S. Uno, T. Abe, I. Adachi, K. Adamczyk and S. Ahn, Belle II technical design report, arXiv:1011.0352.

[2] S. Aoki, Y. Aoki, C. Bernard, T.Blum, G. Colangelo, M.DellaMorte et al., Review of lattice results concerning low-energy particle physics, European Physical Journal C 74 (2014) 1-179, [arXiv:1011.4408].

[3] S. Aoki, Y.Aoki, D.Bečirević, C.Bernard, T.Blum, G. Colangelo et al., Review of lattice results concerning low-energy particle physics, European Physical Journal C 77 (2017) 1-228, [arXiv:1011.4408].

[4] R. J. Dowdall, C. T. H. Davies, R. R. Horgan, C. J. Monahan and J. Shigemitsu, B-meson decay constants from improved lattice nonrelativistic $Q C D$ with physical $u, d, s$, and c quarks, Physical Review Letters 110 (2013) 1-5, [arXiv:1302.2644].

[5] N. H. Christ, J. M. Flynn, T. Izubuchi, T. Kawanai, C. Lehner, A. Soni et al., B -meson decay constants from 2+1 -flavor lattice QCD with domain-wall light quarks and relativistic heavy quarks, Physical Review D - Particles, Fields, Gravitation and Cosmology 91 (2015) 1-24.

[6] O. Witzel, B -meson decay constants with domain-wall light quarks and nonperturbatively tuned relativistic b-quarks, in LATTICE 2013, pp. 0-6, 2013. 1311 .0276v2. 
[7] Y. Aoki, N. Christ, J. Flynn, T. Izubuchi, C. Lehner, M. Li et al., Nonperturbative tuning of an improved relativistic heavy-quark action with application to bottom spectroscopy, Physical Review D 86 (2012) 116003, [arXiv:1206.2554].

[8] Y. Aoki, T. Ishikawa, T. Izubuchi, C. Lehner and A. Soni, Neutral b meson mixings and b meson decay constants with static heavy and domain-wall light quarks, Physical Review D 91 (2015) 114505.

[9] H. Na, C. Monahan, C. Davies, R. Horgan, G. Lepage and J. Shigemitsu, B and B_\{s\} meson decay constants from lattice QCD, Physical Review D 86 (2012) 1-11, [arXiv: 1202.4914].

[10] W. Bietenholz, V. Bornyakov, M. Göckeler, R. Horsley, W. G. Lockhart, Y. Nakamura et al., Flavor blindness and patterns of flavor symmetry breaking in lattice simulations of up, down, and strange quarks, Physical Review D - Particles, Fields, Gravitation and Cosmology 84 (2011) 1-43, [arXiv:1102.5300].

[11] V. G. Bornyakov, R. Horsley, R. Hudspith, Y. Nakamura, H. Perlt, D. Pleiter et al., Wilson flow and scale setting from lattice QCD, arXiv:1508.05916.

[12] Z. Sroczynski, First results from the asymmetric $O(a)$ improved Fermilab action, Nuclear Physics $B$ (Proceedings Supplement) 84 (1999) 971-973, [9910004].

[13] H. W. Lin and N. Christ, Nonperturbatively determined relativistic heavy quark action, Physical Review D - Particles, Fields, Gravitation and Cosmology 76 (2007) 1-20, [0 60800 5].

[14] V. G. Bornyakov, R. Horsley, Y. Nakamura, H. Perlt, D. Pleiter, P. E. L. Rakow et al., Flavour breaking effects in the pseudoscalar meson decay constants, Physics Letters B 767 (2017) 366-373, [arXiv:1612.04798].

[15] P. E. Shanahan, R. Horsley, Y. Nakamura, D. Pleiter, P. E. L. Rakow, G. Schierholz et al., Electric form factors of the octet baryons from lattice QCD and chiral extrapolation, Physical Review D Particles, Fields, Gravitation and Cosmology 90 (2014) 1-17, [arXiv: 1401 . 5862].

[16] Y. Nakamura and H. Stüben, BQCD - Berlin quantum chromodynamics program, Proceedings of Science Lattice 2010 (2010) 7, [arXiv:1011.0199].

[17] P. A. Boyle, The BAGEL assembler generation library, Comput. Phys. Commun. 180 (2009) .

[18] R. G. Edwards and B. Joó, The Chroma software system for lattice QCD, Nuclear Physics B Proceedings Supplements 140 (2005) 832-834, [0 409003 ]. 